\title{
DISTRIBUTION OF PYROPHOSPHATIDIC ACID IN YEAST SPECIES
}

\author{
SHOJI GOTO, SATOSHI HORIGUCHI, HIROSHI KANEKO, * \\ AND TOSHIHIRO ITOH* \\ Institute of Enology and Viticulture, Yamanashi University, \\ Kofu 400, Japan \\ * Division of Chemistry, College of Liberal Art and Sciences, \\ Kitasato University, Sagamihara 228, Japan \\ (Received February 16, 1988)
}

\begin{abstract}
The presence of pyrophosphatidic acid (pyro-PA) was investigated in the lipid extracts of yeasts (241 strains belonging to 167 species of 43 genera). Pyro-PA can be clearly separated from other polar lipids by twodimensional thin layer chromatography, and it can be distinguished from other phospholipids by its specific staining (purple blue) with Dittmer reagent. Pyro-PA was found exclusively in basidiomycetous species (127 strains belonging to 78 species of 14 genera). In contrast, no detectable amount of pyro-PA was found in the cellular lipids of ascomycetous species (114 strains belonging to 89 species of 31 genera). These results demonstrate that the determination of the presence of pyro-PA in the cellular lipids is useful in distinguishing ascomycetous from basidiomycetous yeasts.
\end{abstract}

Ascomycetous and basidiomycetous yeasts have been discriminated by characteristics of cell wall structure (1-4), Diazonium Blue B (DBB) staining (4-6), urease activity $(7,8)$ and the GC content of DNA (9-12). Ascomycetous yeasts have twolayered cell walls, are not stained by $\mathrm{DBB}$, usually lack extracellular urease, and have about 30 to $50 \mathrm{~mol}^{\circ}$ o GC content. Basidiomycetous yeasts have laminar cell walls, stain with DBB, usually produce extracellular urease, and have about 50 to $70 \mathrm{~mol}^{\%} \mathrm{o}$ GC content.

ITOH and KANEKO(13) reported that pyrophosphatidic acid $[P, P$-bis-(1,2diacyl-sn-glycero-3)-pyrophosphate] (pyro-PA), was first found in the cellular lipid of Cryptococcus neoformans (13), and was also found in 2 strains of Cryptococcus, 2 of Rhodotorula, and 1 of Trichosporon $(14,15)$. This paper describes the distribution of pyro-PA in ascomycetous and basidiomycetous yeast species (241 strains

Address reprint requests to: Dr. S. Goto, Institute of Enology and Viticulture, Yamanashi University, Kofu 400, Japan. 
belonging to 167 species of 43 genera), and suggests that the presence of pyro-PA in yeast species is significant in yeast taxonomy.

\section{MATERIALS AND METHODS}

Yeast strains used. The strains used in this study (167 species of 43 genera) are listed in Table 1.

Growth and harvest of yeast cells. Yeasts were inoculated on YM agar plates ( $10 \mathrm{~g}$ of glucose, $3 \mathrm{~g}$ of yeast extract, $3 \mathrm{~g}$ of malt extract, $10 \mathrm{~g}$ of peptone and $20 \mathrm{~g}$ of agar in $1,000 \mathrm{ml}$ of tap water) and grown for 48 to $96 \mathrm{hr}$ at 18 to $22^{\circ} \mathrm{C}$. The cells were harvested after they spread over all of the surface of the plate (14).

Lipid extraction. Lipids were extracted from fresh yeast cells according to ITOH and Kaneko(16). Five to $10 \mathrm{~g}$ of the wet cells was suspended in $15 \mathrm{ml}$ of a chloroform : methanol $(1: 2 \mathrm{v} / \mathrm{v})$ mixture containing $10 \mathrm{~g}$ of glass beads $(1.0 \mathrm{~mm}$ diameter). The mixture was homogenized for $30 \mathrm{~min}$ in a cell homogenizer (mixtower A-14, Taiyou). The disrupted cell suspension was filtered through a glass filter (4G). The residual cell debris was suspended in $50 \mathrm{ml}$ of a chloroform : methanol $(2: 1 \mathrm{v} / \mathrm{v})$ mixture and stirred with a magnetic stirrer for $30 \mathrm{~min}$ at room temperature under an atmosphere of nitrogen, then filtered. The stirring and filtering processes were repeated three more times. The combined chloroform-methanol filtrate was washed with $0.9 \% \mathrm{NaCl}$ solution to remove the non-lipid contaminants according to FolCH's method (17).

Thin layer chromatography (TLC). The TLC methods described by ITOH and Kaneko (16) were used with Silica gel precoated plates (Merck Art. 5721, $10 \times 10 \mathrm{~cm})$. The following solvent mixtures were used: first dimension, chloroform : methanol : water $(65: 25: 4)$; second dimension, chloroform : methanol : $7 \mathrm{~N}$ $\mathrm{NH}_{4} \mathrm{OH}(65: 35: 5 \mathrm{v} / \mathrm{v})$. The following detection reagents were used; the polar lipids were detected by spraying Dittmer reagent and charring at $150^{\circ} \mathrm{C}$ for $30 \mathrm{~min}$. PyroPA was clearly separated from other polar lipids on the two-dimensional TLC, and distinguished from other phospholipids by specific staining purple blue with Dittmer reagent. Therefore, the presence of pyro-PA in a lipid sample was determined primarily by two-dimensional TLC. When a trace amount of pyro-PA was detected, the sample was concentrated for further chromatography as follows. The lipid-fraction was extracted with a chloroform : methanol $(2: 1 \mathrm{v} / \mathrm{v})$ mixture from the spot corresponding to pyro-PA on the chromatograms of the crude total lipids. After this the lipid fraction was dried and the occurrence of pyro-PA was confirmed by two-dimensional TLC as described above.

DBB test. The Diazonium Blue B (DBB) color test was performed as described by VAN Der Walt and Hopsu-Havu (5). Cultures were plated on YM agar for 2 weeks at $24^{\circ} \mathrm{C}$, or $20^{\circ} \mathrm{C}$ in the case of psychrophilic strains. The reagent was prepared immediately before use by dissolving the DBB salt $(1 \mathrm{mg} / \mathrm{ml}$, Fast Blue Salt B, Tokyo Chemical Ind. Co., Tokyo) in chilled $0.1 \mathrm{M}$ Tris- $\mathrm{HCl}$ buffer ( $\mathrm{pH}$ 7.0). The chilled DBB (1 to 2 drops) was applied directly to the surface of colonies, 
and the color reaction was recorded. The alkaline-ethanol procedure was applied to the red yeast strains according to the method described by HAGLER and AHEARN (6). The strains were grown in $5.0 \mathrm{ml}$ of Yeast Nitrogen Base with $0.5 \%$ glucose for 5 days at $24^{\circ} \mathrm{C}$. The psychrophilic strains were cultured at $20^{\circ} \mathrm{C}$.

Urease test. Production of urease was determined using Christensen urea agar (Difco), and the results were recorded after 2 and 5 days.

Fermentation test. Fermentation of glucose was observed by the Durham tube method after 1,2 , and 3 weeks at $25^{\circ} \mathrm{C}$ or $20^{\circ} \mathrm{C}$ for psychrophilic strains, Nitrogen Base (Difco) supplemented with $2 \%$ glucose was used.

\section{RESULTS AND DISCUSSION}

Presence of pyro-PA in yeasts other than Candida and Trichosporon

Pyro-PA can be clearly separated from other polar lipids on two-dimensional TLC (Fig. 1), and this can be confirmed by its specific staining-purple blue-with Dittmer reagent. The presence of pyro-PA, DBB coloring, and urease activity in the 150 strains used, other than Candida and Trichosporon, are shown in Tables 2, 3, and 4.

Pyro-PA was found in 33 strains belonging to 18 species in the 6 genera of basidiomycetous yeast species (Strain No. B 01-B 33) Cystofilobasidium, Filobasidiella, Filobasidium, Leucosporidium, Rhodosporidium, and Sporidiobolus, and in 55 strains belonging to 35 species of the 6 genera of basidiomycetous anamorphs Bullera, Cryptococcus, Phaffia, Rhodotorula, Sporobolomyces, and Sterigmatomyces. In Rhodosp. malvinellum (B 23 and B 24), B. alba (I 31), B. tsugae (I 38), and $S p$. roseus (I 79) the presence of pyro-PA was confirmed by rechromatography. In contrast, pyro-PA was not detected in ascosporogenous yeast species ( 34 strains belonging to 28 species of 23 genera (A 01-A 35)) nor in 28 strains belonging to 18 species of the 6 genera of ascomycetous anamorphs (I 01-I 28) Brettanomyces, Kloeckera, Myxozyma, Oosporidium, Saitoella, and Trigonopsis. ITOH and KANEKO (14) reported that pyro-PA was detected in Kl. apiculata, but this phospholipid was not found in the same strain in the following study (15). In this study, pyro-PA was not found in 3 strains of $\mathrm{Kl}$. apiculata, 10 strains belonging to 4 species of Kloeckera, and 6 strains belonging to 4 species of its teleomorph, Hanseniaspora. Two strains of Myxozyma (I 23 and I 24) were formerly identified as Torulopsis melibiosum and Candida mucilagina $(18,19)$. They were established $(20)$ on the basis of their production of extracellular polysaccharide, having a twolayered structure of cell walls $(20,21)$ and carbohydrate composition of intact cells (mannan and glucan as dominant components) (20). From those characteristics, this genus was considered to be ascomycetous. Pyro-PA was not detected in 2 strains of Myxozyma melibiosi (I 23) and Myxozyma mucilagina (I 24). Saitoella complicata(22) was formerly identified as Rhodotorula glutinis(23). This yeast was identified as ascomycetous anamorph on the basis of having two-layered of cell walls and a negative reaction to DBB. Pyro-PA was not found in the two strains of 
Table 1. The yeast strains used in this study.

\begin{tabular}{|c|c|c|}
\hline $\begin{array}{c}\text { Strain } \\
\text { No. }\end{array}$ & Yeasts & Sources \\
\hline A 01 & Ambrosiozyma platypodis (Baker et Kreger-van Rij) van der Walt & IFO 1471 \\
\hline A 02 & Citeromyces matritensis (Santa-Maria) Santa Maria & IFO 0954 \\
\hline A 03 & Debaryomyces hansenii (Zopf) Lodder et Kreger-van Rij & IAM 4978 \\
\hline A 04 & Dekkera bruxellensis van der Walt & CBS $74^{\mathrm{T}}$ \\
\hline A 05 & Hanseniaspora osmophila (Niehaus) Phaff, Miller et Shifrine & CBS $313^{\mathrm{T}}$ \\
\hline A 06 & & AJ 5186 \\
\hline A 07 & uvarum (Niehaus) Shehata, Mrak et Phaff & AJ 5184 \\
\hline A 08 & valbyensis Kloecker & CBS $479^{\mathrm{T}}$ \\
\hline A 09 & & AJ 5181 \\
\hline A 10 & vineae van der Walt et Tschenschner & CBS 2171 \\
\hline A 11 & Hansenula anomala (Hansen) H. et Sydow & RY I-2-1 \\
\hline A 12 & Issatchenkia terricola (van der Walt) Kurtzmen, Smiley et Johnson & RY 4024 \\
\hline A 13 & Kluyveromyces phaffi (van der Walt) van der Walt & CBS 4417 \\
\hline A 14 & Lipomyces starkeyi Lodder et Kreger-van Rij & CBS 1807 \\
\hline A 15 & Lodderomyces elongisporus (Recca et Mrak) van der Walt & AJ 5470 \\
\hline A 16 & Metschnikowia pulcherrima (Metschnikoff) Kamienski & RY K-427 \\
\hline A 17 & Nadsonia elongata Konokotina & IFO 0665 \\
\hline A 18 & fluvescens (Nadson et Konokotina) Sydow & IFO 0666 \\
\hline A 19 & Pachysolen tannaphilus Boidin et Adzet & AJ 4282 \\
\hline A 20 & Pichia membranaefaciens Hansen & IAM 4987 \\
\hline A 21 & & RY IV-5-1 \\
\hline A 22 & Saccharomyces cerevisiae Meyen et Hansen & $\operatorname{CBS} 1171^{\mathrm{T}}$ \\
\hline A 23 & & RY W-3 \\
\hline A 24 & Saccharomycodes ludwigii Hansen & IFO 1043 \\
\hline A 25 & & RY K-511 \\
\hline A 26 & Saccharomycopsis capsularis Schionning & IAM 4307 \\
\hline A 27 & Schizosaccharomyces malidevorans Rankine et Fornachon & AWRI 442 \\
\hline A 28 & Schizosaccharomyces pombe Lindner & RY No. 1011 \\
\hline A 29 & & RY No. 1012 \\
\hline A 30 & Sporopachdermia lactativola Rodrigues de Miranda & CCY $17-12-1$ \\
\hline A 31 & Torulaspora delbrueckii (Lindner) Lindner & IAM 12236 \\
\hline A 32 & Zygosaccharomyces rouxii (Boutroux) Yarrow & NRRL 2547 \\
\hline A 33 & Wickerhamia fluorescens Soneda & AJ 4285 \\
\hline A 34 & Yarrowia lipolytica (Wickerham et al.) van der Walt et von Arx & CBS 6303 \\
\hline В 01 & Cystofilobasidium capitatum (Fell, Hunter et Tallman) Oberw. et Bandoni & ATCC $24507^{\mathrm{T}}$ \\
\hline В 02 & & ATCC 24508 \\
\hline В 03 & Filobasidiella neoformans Kown-Chung var. neoformans & $\mathrm{NIH} 12^{\mathrm{T}}$ \\
\hline В 04 & var. bacillispora & NIH 191 \\
\hline \multirow[t]{2}{*}{ В 05} & Filobasidium capsuligenum (Fell, Statzell, Hunter et Phaff) & \\
\hline & Rodrigues de Miranda & IFO 1119 \\
\hline В 06 & floriforme Olive & CBS $6241^{\mathrm{T}}$ \\
\hline В 07 & uniguttulatum Kown-Chung & $\mathrm{CU}-1$ \\
\hline В 08 & Leucosporidium frigidum Fell, Statzell, Hunter et Phaff & IFO 1851 \\
\hline В 09 & nivalis Fell, Hunter et Phaff & IFO 1852 \\
\hline B 10 & scottii Fell, Statzell, Hunter et Phaff & IFO 1287 \\
\hline B 11 & Rhodosporidium dacryoidum Fell, Hunter et Tallman & CBS $6353^{\mathrm{T}}$ \\
\hline B 12 & diobovatum Newell et Hunter & IFO 0667 \\
\hline B 13 & & RY FC 16 \\
\hline
\end{tabular}


Table 1. (continued)

\begin{tabular}{|c|c|c|}
\hline $\begin{array}{l}\text { Strain } \\
\text { No. }\end{array}$ & Yeasts & Sources \\
\hline B 14 & & RY No. 231 \\
\hline B 15 & & RY K-1-1 \\
\hline B 16 & & IAM 12259 \\
\hline B 17 & & IFO 1828 \\
\hline B 18 & & IAM $12260^{\mathrm{T}}$ \\
\hline B 19 & Rhodosporidium infirmominiatum Fell, Hunter et Tallman & CBS 6962 \\
\hline B 20 & & IFO 1057 \\
\hline B 21 & & CBS 6350 \\
\hline B 22 & & CBS 6352 \\
\hline B 23 & malvinellum Fell et Hunter & CCY $62-4-1^{\mathrm{T}}$ \\
\hline B 24 & & CCY $62-4-2$ \\
\hline B 25 & sphaerocarpum Newell et Fell & IAM 12262 \\
\hline B 26 & & CCY 62-1-1 \\
\hline B 27 & toruloides Banno & IFO $0559^{\mathrm{T}}$ \\
\hline B 28 & & CCY 62-3-1 \\
\hline B 29 & & CCY $62-3-2$ \\
\hline B 30 & Sporidiobolus johansonii Nyland & IFO 6903 \\
\hline B 31 & pararoseus Fell et Tallman & IFO 0376 \\
\hline B 32 & ruinenii Holzschu, Tredick et Phaff & IFO 1689 \\
\hline B 33 & salmonocolor Fell et Tallman & CBS 6832 \\
\hline I 01 & Brettanomyces abstinens Yarrow et Ahearn & CBS $6055^{\mathrm{T}}$ \\
\hline I 02 & anomalus Custers & CBS $77^{\mathrm{T}}$ \\
\hline I 03 & bruxellensis Kufferath et van Laer & CBS $72^{\mathrm{T}}$ \\
\hline I 04 & claussenii Custers & CBS $76^{\mathrm{T}}$ \\
\hline I 05 & custersianus van der Walt & CBS $4805^{\mathrm{T}}$ \\
\hline I 06 & $\begin{array}{l}\text { intermedius (Krumbholz et Tauschanoff) van der Walt } \\
\text { et van Kerken }\end{array}$ & $\operatorname{CBS} 73^{\mathrm{T}}$ \\
\hline I 07 & lambicus Kufferath et van Laer & CBS $75^{\mathrm{T}}$ \\
\hline I 08 & naardenensis Kalfschoten et Yarrow & CBS $6042^{\mathrm{T}}$ \\
\hline I 09 & Kloeckera africana (Klocker) Janke & CBS $277^{\mathrm{T}}$ \\
\hline I 10 & & AJ 5173 \\
\hline I 11 & & RY K-415 \\
\hline I 12 & apiculata (Reess emend. Klocker) Janke & CBS $104^{\mathrm{T}}$ \\
\hline I 13 & & AJ 4806 \\
\hline I 14 & & RY 00-1 \\
\hline I 15 & corticis (Klocker) Janke & AJ 5171 \\
\hline I 16 & & AJ 5198 \\
\hline I 17 & & CBS $106^{\mathrm{T}}$ \\
\hline I 18 & & RY 983 \\
\hline I 19 & & RY 991 \\
\hline I 20 & japonica Saito et Ohtani & CBS $281^{\mathrm{T}}$ \\
\hline I 21 & & AJ 5469 \\
\hline I 22 & javanica (Klocker) Janke & RY 29B \\
\hline I 23 & $\begin{array}{c}\text { Myxozyma melibiosi (Shifrine et Phaff) van der Walt, Weijman } \\
\text { et von Arx }\end{array}$ & CBS 2102 \\
\hline I 24 & $\begin{array}{l}\text { mucilagina (Ohaff, Starmer, Mirand et Miller) van der Walt, } \\
\text { Weijman et von Arx }\end{array}$ & UCD $76-236 \mathrm{C}$ \\
\hline I 25 & Oosporidium margaririferum Stautz & IFO 1208 \\
\hline
\end{tabular}


Table 1. (continued)

\begin{tabular}{|c|c|c|}
\hline $\begin{array}{c}\text { Strain } \\
\text { No. }\end{array}$ & Yeasts & Sources \\
\hline I 26 & Saitoella complicata Goto, Sugiyama, Hamamoto et Komagata & RY H-3-9-1 \\
\hline I 27 & & RY H-3-9-2 \\
\hline I 28 & Trigonopsis variabilis Schachner & IAM 4994 \\
\hline I 31 & Bullera alba (Hanner) Derx & *K No. 85 \\
\hline I 32 & & ${ }^{*} \mathrm{~K}$ No. 92 \\
\hline I 33 & & ${ }^{*} \mathrm{~K}$ No. 101 \\
\hline I 34 & & ${ }^{*} \mathrm{~K}$ No. 105 \\
\hline I 35 & dendrophila van der Walt et Scott & CBS $6074^{\mathrm{T}}$ \\
\hline I 36 & piricola Stadelmann & CCY $12-3-2$ \\
\hline I 37 & singularis (Phaff et do Carmo-Sousa) Rodrigues de Miranda & CBS $5109^{\mathrm{T}}$ \\
\hline I 38 & tsugae Phaff et do Carmo-Sousa & CCY 12-2-1 \\
\hline I 39 & Cryptococcus albidus (Saito) var. albidus & IFO 1320 \\
\hline I 40 & var. aerius (Saito) Phaff et Fell & IAM 4317 \\
\hline I 41 & ater Castellani et Cooke & CBS $4685^{\mathrm{T}}$ \\
\hline I 42 & bhutanensis Goto et Sugiyama & AJ 5129 \\
\hline I 43 & gastricus Reiersol et di Menna & CCY 17-5-1 \\
\hline I 44 & heveanensis (Groenewege) Baptist et Kurtzman & CBS 569 \\
\hline I 45 & himalayensis Goto et Sugiyama & RY H-3-11-6 \\
\hline I 46 & kuetzingii Fell et Phaff & CCY 17-11-1 \\
\hline I 47 & laurentii (Kufferath) Skinner & RY EC $2-3$ \\
\hline I 48 & luteolus (Saito) Skinner & IAM 12207 \\
\hline I 49 & macerans (Frederiksen) Phaff et Fell & CCY 17-19-1 \\
\hline I 51 & neoformans (Sanfelice) Vuillemin & IAM 12253 \\
\hline I 52 & skinneri Phaff et do Carmo-Sousa & CCY 17-17-1 \\
\hline I 53 & terreus di Menna & CCY 17-8-1 \\
\hline I 54 & Phaffia rhogozyma Miller, Yoneyama et Soneda & CBS 5905 \\
\hline I 55 & Rhodotorula araucariae Grinbergs et Yarrow & CBS $6031^{\mathrm{T}}$ \\
\hline I 56 & aurantiaca (Saito) Lodder & RY EC 7-4 \\
\hline I 57 & fujisanensis (Soneda) Johnson et Phaff & CBS $4551^{\mathrm{T}}$ \\
\hline I 58 & & RY No. 870 \\
\hline I 59 & glutinis (Fresenis) Harrison & IAM 4988 \\
\hline I 60 & & IAM 12263 \\
\hline I 61 & & IFO 0697 \\
\hline I 62 & & IFO 1099 \\
\hline I 63 & & RY KC 4-4 \\
\hline I 64 & & RY KC 9 \\
\hline I 65 & & IAM 12228 \\
\hline I 66 & & CBS $20^{\mathrm{T}}$ \\
\hline \multirow[t]{2}{*}{ I 67} & glutinis (Fres.) Harrison var. salinaria Hirosawa & \\
\hline & et Takada & IFO 1438 \\
\hline I 68 & graminis di Menna & IFO 0190 \\
\hline I 69 & & CCY $20-14-1^{\mathrm{T}}$ \\
\hline I 70 & lactosa Hasegawa & IAM $12230^{\mathrm{T}}$ \\
\hline I 71 & minuta (Saito) Harrison & IFO $0387^{\mathrm{T}}$ \\
\hline I 72 & & CBS 5475 \\
\hline I 73 & rubra (Demme) Lodder & ATCC $9449^{\mathrm{T}}$ \\
\hline I 74 & Sporobolomyces albo-rubescens Derx & CBS $482^{\mathrm{T}}$ \\
\hline I 75 & gracilis Derx & IFO $1227^{\mathrm{T}}$ \\
\hline
\end{tabular}


Table 1. (continued)

\begin{tabular}{|c|c|c|}
\hline $\begin{array}{c}\text { Strain } \\
\text { No. }\end{array}$ & Yeasts & Sources \\
\hline I 76 & Sporobolomyces hispanicus Pelaez et Ramirez & CBS 497 \\
\hline I 77 & odorus Derx & ${ }^{*} \mathrm{~K}$ No. 19 \\
\hline I 78 & roseus Kluyver et van Niel & ${ }^{*} \mathrm{~K}$ No. 69 \\
\hline I 79 & & ${ }^{*} \mathrm{~K}$ No. 70 \\
\hline I 80 & & ${ }^{*} \mathrm{~K}$ No. 71 \\
\hline I 81 & & ${ }^{*} \mathrm{~K}$ No. 72 \\
\hline I 82 & & ${ }^{*} \mathrm{~K}$ No. 73 \\
\hline I 84 & salmonicolor (Fisher et Brebeck) Kluyver et van Niel & ${ }^{*} \mathrm{~K}$ No. 7 \\
\hline I 85 & shibatanus (Okunuki) Verona et Ciferri & ${ }^{*} \mathrm{~K}$ No. 56 \\
\hline I 86 & & ${ }^{*} \mathrm{~K}$ No. 75 \\
\hline I 87 & Sterigmatomyces elviae Sonk et Yarrow & IFO $1843^{\mathrm{T}}$ \\
\hline C 01 & Candida acutus Goto & RY C-96-2 \\
\hline $\mathrm{C} 02$ & álbicans (Robin) Berkhout & IAM 4966 \\
\hline C 03 & amylolenta vander Walt, Scott et van der Klift & CBS $6039^{\mathrm{T}}$ \\
\hline C 04 & $\begin{array}{l}\text { antarctica (Goto, Sugiyama et Iizuka) Kurtzman, Smiley, } \\
\text { Johnson et Hoffman }\end{array}$ & CBS 6821 \\
\hline $\mathrm{C} 05$ & aquatica Jones et Slooff & CBS $5443^{\mathrm{T}}$ \\
\hline C 06 & auriculariae (Nakase) Meyer et Yarrow & IFO 1580 \\
\hline $\mathrm{C} 07$ & auringiensis Santa Maria & CBS 6913 \\
\hline C 08 & bacarum (Buhagiar) Meyer et Yarrow & CBS $6526^{\mathrm{T}}$ \\
\hline C 09 & blankii Buckley et van der Uden & CBS $1898^{\mathrm{T}}$ \\
\hline C 10 & boidinii Ramirez & AJ 4779 \\
\hline C 11 & bombicola (Spencer, Gorin et Tulloch) Meyer et Yarrow & ATCC 22214 \\
\hline C 12 & buffonii (Ramirez) van Uden et Buckley & CBS $2838^{\mathrm{T}}$ \\
\hline C 13 & catenulata Diddens et Lodder & IAM 12182 \\
\hline C 14 & chiropterorum Grose et Marinkelle & CBS $6064^{\mathrm{T}}$ \\
\hline C 15 & colliculosa (Hartmann) Meyer et Yarrow & IAM 4426 \\
\hline C 16 & curiosa Komagata et Nakase & IFO 1336 \\
\hline C 17 & curvata (Diddens et Lodder) Lodder et Kreger-van Rij & IFO 1159 \\
\hline C 18 & diffluens Ruinen & CBS $5233^{\mathrm{T}}$ \\
\hline C 19 & & IFO 1521 \\
\hline C 20 & edax van der Walt et Nel & CBS $5657^{\mathrm{T}}$ \\
\hline C 21 & famata (Harrison) Meyer et Yarrow & IFO 0623 \\
\hline C 22 & & IFO 0380 \\
\hline C 23 & & AJ 4385 \\
\hline C 24 & foliorum Ruinen & CBS 6370 \\
\hline C 25 & fusiformata Buhagiar & CBS 6951 \\
\hline C 26 & guilliermondii (Castellani) Langeron et Guerra & IAM 4974 \\
\hline C 27 & hellenica (Verona et Picci) King et Jong & CBS $4099^{\mathrm{T}}$ \\
\hline C 28 & humicola (Daszewska) Diddens et Lodder & IFO 0760 \\
\hline C 29 & & IFO 1527 \\
\hline C 30 & & AJ 4385 \\
\hline C 31 & hydrocarbofumaria Yamada et al. ex Ramirez & IFO 1973 \\
\hline C 32 & hylophila van der Walt, van der Klift et Scott & CBS $6226^{\mathrm{T}}$ \\
\hline C 33 & ingeniosa (di Menna) Meyer et Yarrow & AJ 5002 \\
\hline C 34 & inositophila Nakase & IFO 1575 \\
\hline C 35 & intermedia (Ciferri et Ashford) Lahgeron et Guerra & IAM 12185 \\
\hline C 36 & javanica Ruinen & CBS 5236 \\
\hline
\end{tabular}


Table 1. (continued)

\begin{tabular}{|c|c|c|}
\hline $\begin{array}{c}\text { Strain } \\
\text { No. }\end{array}$ & Yeasts & Sources \\
\hline C 37 & Candida krissii Goto, Yamasato et Iizuka & CBS $6519^{\mathrm{T}}$ \\
\hline \multirow[t]{2}{*}{ C 38} & kruisii (Kockova-Kratochvilova et Ondrusova) & \\
\hline & Meyer et Yarrow & ATCC 52828 \\
\hline C 39 & krusei (Castellani) Berkhout & RY WF 16 \\
\hline $\mathrm{C} 40$ & lambica (Lindner et Genoud) van Uden et Buckley & AJ 4461 \\
\hline C 41 & lusitaniae van Uden et do Carmo-Sousa & IAM 12189 \\
\hline C 42 & magnoliae (Lodder et Kreger-van Rij) Meyer et Yarrow & IFO 0661 \\
\hline C 43 & maltosa Komagata, Nakase et Katsuya & IAM 12247 \\
\hline $\mathrm{C} 44$ & mamillae Goto & RY PJ 819 \\
\hline C 45 & mucilagina Phaff, Starmer, Miranda et Miller & CBS $7071^{\mathrm{T}}$ \\
\hline C 46 & muscorum di Menna & CBS $6921^{\mathrm{T}}$ \\
\hline C 47 & parapsilosis (Ashford) Langeron et Talice & IAM 12270 \\
\hline $\mathrm{C} 48$ & & AJ 4575 \\
\hline \multirow[t]{2}{*}{ C 49} & philyla (van der Walt, van der Klift et Scott) & \\
\hline & Meyer et Yarrow & CBS $6272^{\mathrm{T}}$ \\
\hline C 50 & podzolica Babjeva et Reshetova & CBS $6819^{\mathrm{T}}$ \\
\hline C 51 & psychrophila (Goto, Sugiyama et Iizuka) Meyer et Yarrow & CBS $5956^{\mathrm{T}}$ \\
\hline C 52 & pustula (Buhagiar) Meyer et Yarrow & CBS $6527^{\mathrm{T}}$ \\
\hline C 53 & rugosa (Anderson) Diddens et Lodder & IAM 12198 \\
\hline C 54 & sake (Saito et Ota) van Uden et Buckley & IAM 12200 \\
\hline C 55 & sonckii Hopsu-Haru, Tunnela et Yarrow & CBS 8115 \\
\hline C 55 & sonckii Hopsu-Havu, Tunnela et Yarrow & CBS 8115 \\
\hline C 57 & stellata (Kroemer et Krumbholz) Meyer et Yarrow & AJ 4394 \\
\hline C 58 & terebra Sugiyama et Goto & RY KC-1-2 \\
\hline C 59 & tropicalis (Castellani) Berkhout & IAM 4185 \\
\hline C 60 & & RY E-16 \\
\hline C 61 & tsukubaensis Onishi & RY No. 668 \\
\hline C 62 & utilis (Henneberg) Lodder et Kreger-van Rij & IAM 4961 \\
\hline C 63 & valdiviana Grinberg et Yarrow & CBS 5721 \\
\hline C 64 & valida (Leberle) van Uden et Buckley & IAM 12190 \\
\hline C 65 & & RY K-504 \\
\hline C 66 & versatilis (Etchells et Bell) Meyer et Yarrow & IAM 4993 \\
\hline C 67 & zeylanoides (Castellani) Langeron et Guerra & IAM 12204 \\
\hline Т 01 & Trichosporon aculeatum Phaff, Miller et Shifrine & ATCC 15540 \\
\hline Т 02 & & ATCC 28689 \\
\hline Т 03 & aquatile Hedrick et Dupont & ATCC $22310^{\mathrm{T}}$ \\
\hline Т 04 & brassicae Nakase & $* *$ SH 280 \\
\hline Т 05 & capitatum Diddens et Lodder & **SH 233 \\
\hline Т 06 & & ** SH 281 \\
\hline Т 07 & cutaneum (de Beurm., Gougerot et Vaucher) Ota & CBS $4829^{\mathrm{T}}$ \\
\hline Т 08 & & CBS 7065 \\
\hline Т 09 & & IFO 1200 \\
\hline $\mathrm{T} 10$ & & ${ }^{* *} \mathrm{SH} 285$ \\
\hline $\mathrm{T} 11$ & & **SH 287 \\
\hline Т 12 & & ${ }^{* *} \mathrm{SH} 418$ \\
\hline T 13 & & ${ }^{* *} \mathrm{SH} 422$ \\
\hline T 14 & & ATCC 24659 \\
\hline T 15 & & ATCC 34396 \\
\hline
\end{tabular}


Table 1. (continued)

\begin{tabular}{ccc}
\hline $\begin{array}{c}\text { Strain } \\
\text { No. }\end{array}$ & Yeasts & Sources \\
\hline T 16 & Trichosporon fennicum Sonck et Yarrow & CBS 5928 \\
T 17 & fermentans Diddens & $* *$ SH 292 \\
T 18 & & $*$ SH 428 \\
T 19 & melibiosaceum Scott et van der Walt & $*$ SH 429 \\
T 20 & pullulans (Lindner) Diddens et Lodder & CBS 6087 \\
T 21 & & $*$ SH 293 \\
T 22 & & $*$ SH 316 \\
T 23 & & $* *$ SH 317 \\
T 24 & & $* *$ SH 318 \\
\hline
\end{tabular}

Abbreviations: IFO, Institute for Fermentation, Osaka, Japan; IAM, Institute of Applied Microbiology, The University of Tokyo, Japan; CBS, Centraalbureau voor Schimmelcultures, Delft, The Netherlands; AJ, Central Research Laboratories, Ajinomoto Co., Inc., Kawasaki, Japan; RY, Laboratory of Applied Microbiology, Institute of Enology and Viticulture, Yamanashi University, Kofu, Japan; AWRI, Australian Wine Research Institute, Adrade, Australia; CCY, Czechoslovak Collection of Yeasts, Bratislava, Czechoslovakia; ATCC, American Type Culture Collection, Rockville, Md., U.S.A.; NRRL, ARS Culture Collection, Northern Regional Research Center, Peoria, U.S.A.; NIH, National Institutes of Health, Bethesda, Md., U.S.A.

${ }^{*} \mathrm{~K}$ strains accepted an offer from Prof. Dr. K. Komagata, Institute of Applied Microbiology, The University of Tokyo, Tokyo, Japan and ${ }^{* *} \mathrm{SH}$ strains accepted an offer from Prof. Dr. T Sutoh, Nippon Veterinary and Zootechnical College, Musashino, Tokyo, Japan.

$\mathrm{T}$ : type strain.
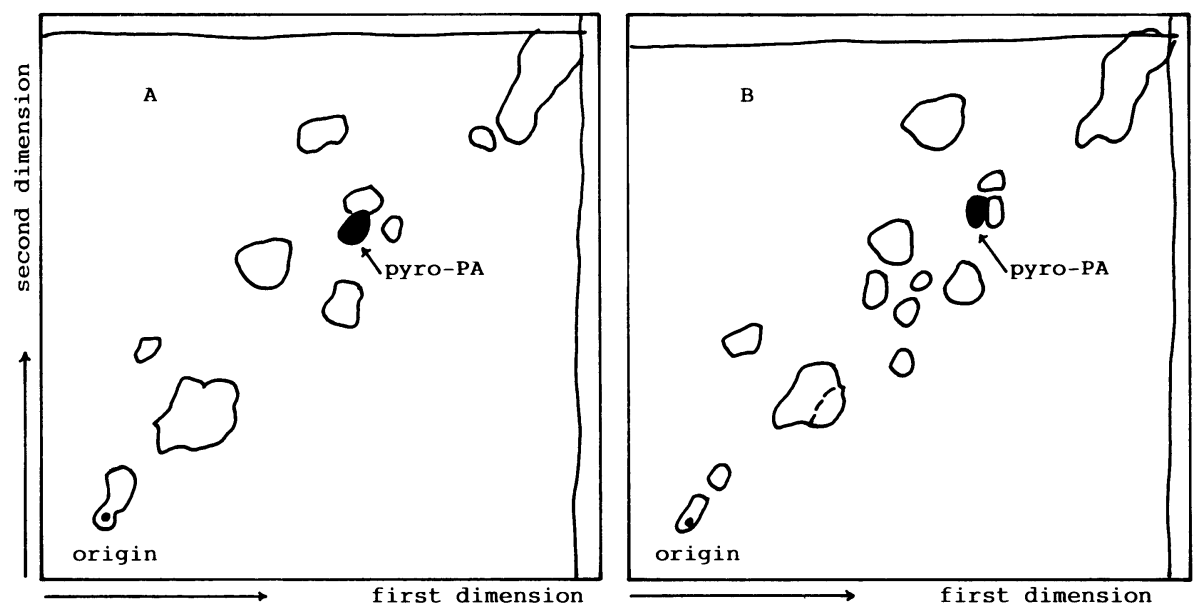

Fig. 1. Thin layer chromatograms: (A) the polar lipids of Rhodosp. toruloides IFO $0559^{\mathrm{T}}$ (B 27); (B) the polar lipids of $R$. glutinis CBS $20^{\mathrm{T}}$ (I 66).

Plate: silica gel precoated plate, $10 \times 10 \mathrm{~cm}$ (Merck Art. 5715). Solvent system: first dimension, chloroform:methanol: water $(65: 24: 4 \mathrm{v} / \mathrm{v})$; second dimension, chloroform: methanol: $7 \mathrm{~N}$ ammonium hydroxide $(56: 35: 5 \mathrm{v} / \mathrm{v})$. Detection: by spraying the chromatograms with Dittmer reagent and charring at $160^{\circ} \mathrm{C}$ for $60 \mathrm{~min}$. Pyro-PA= pyrophosphatidic acid. 
Table 2. The occurrence of pyrophosphatidic acid, DBB reaction and urease activity in ascosporogenous yeasts.

\begin{tabular}{|c|c|c|c|c|}
\hline Strain No. & Yeasts & Pyro-PA & DBB & Urease \\
\hline A 01 & A. platypodis & - & - & - \\
\hline A 02 & Cit. matritensis & - & - & + \\
\hline A 03 & Deb. hansenii & - & - & - \\
\hline A 04 & D. bruxellensis & - & - & - \\
\hline A 05 & H'spora osmophila & - & - & - \\
\hline A 06 & & - & - & - \\
\hline A 07 & uvarum & - & - & - \\
\hline A 08 & valbyensis & - & - & - \\
\hline А 09 & & - & - & - \\
\hline A 10 & vineae & - & - & - \\
\hline A 11 & H. anomala & - & - & - \\
\hline A 12 & I. terricola & - & - & + \\
\hline A 13 & K. phaffi & - & - & - \\
\hline A 14 & L. starkeyi & - & - & + \\
\hline A 15 & Lod. elongisporus & - & - & - \\
\hline A 16 & M. pulcherrima & - & - & - \\
\hline A 17 & N. elongata & - & - & + \\
\hline A 18 & fluvescens & - & - & + \\
\hline A 19 & Pa. tannophilus & - & - & - \\
\hline A 20 & P. membranaefaciens & - & - & - \\
\hline A 21 & & - & - & - \\
\hline A 22 & Sacch. cerevisiae & - & - & - \\
\hline A 23 & & - & - & - \\
\hline A 24 & S'codes ludwigii & - & - & - \\
\hline A 25 & & - & - & - \\
\hline A 26 & S. capsularis & - & - & - \\
\hline A 27 & Schiz. malidevorans & - & - & + \\
\hline A 28 & pombe & - & - & + \\
\hline A 29 & pombe & - & - & + \\
\hline A 30 & Sporop. lactativola & - & - & - \\
\hline A 31 & T'spora delbrueckii & _- & - & _- \\
\hline A 32 & Zygosacch. rouxii & - & - & - \\
\hline A 33 & W. fluorescens & - & - & - \\
\hline A 34 & $Y$. lipolytica & - & - & + \\
\hline
\end{tabular}

Saitoella complicata (I 26 and I 27).

Generally, for the yeast species in which pyro-PA was detected, the DBB reaction and urease activity were positive. The species having those characteristics belong to the basidiomycetous yeasts.

The DBB test $(1,3-6,12)$, in conjunction with the cell wall structure, was a practical procedure for discrimination between ascomycetous and basidiomycetous yeasts in which a sexual stage was not demonstrated. However, the simple procedure on solid medium often gave indecisive results, particularly in the coloration of red yeasts such as Rhodosporidium, Rhodotorula, and Sporobolomyces. 
Table 3. The occurrence of pyrophosphatidic acid, DBB reaction and urease activity in basidiosporogenous yeasts.

\begin{tabular}{|c|c|c|c|c|}
\hline Strain No. & Yeasts & Pyro-PA & DBB & Urease \\
\hline В 01 & Cystof. capitatum & + & $\mathrm{v}$ & $\mathrm{w}$ \\
\hline В 02 & & + & $\mathrm{v}$ & w \\
\hline В 03 & Fil. neoformans var. neoformans & + & + & + \\
\hline B 04 & var. bacillispora & + & + & + \\
\hline В 05 & F. capsuligenum & + & + & + \\
\hline В 06 & floriforme & + & + & + \\
\hline В 07 & uniguttulatum & + & + & + \\
\hline В 08 & Leu. frigidum & + & + & + \\
\hline В 09 & nivalis & + & + & + \\
\hline B 10 & scottii & + & + & + \\
\hline B 11 & Rhodosp. dacryoidum & + & + & + \\
\hline B 12 & diobovatum & + & + & + \\
\hline B 13 & & + & $\mathrm{v}$ & + \\
\hline B 14 & & + & + & + \\
\hline B 15 & & + & + & + \\
\hline B 16 & & + & $\mathrm{v}$ & + \\
\hline B 17 & & + & + & + \\
\hline B 18 & & + & + & $\mathrm{w}$ \\
\hline B 19 & infirmominiatum & + & + & + \\
\hline B 20 & & + & + & w \\
\hline B 21 & & + & + & + \\
\hline B 22 & & + & $\mathrm{v}$ & + \\
\hline B 23 & malvinellum & + & $\mathrm{v}$ & $\mathrm{w}$ \\
\hline B 24 & & + & $\mathrm{v}$ & - \\
\hline B 25 & sphaerocarpum & + & $\mathrm{v}$ & + \\
\hline B 26 & & + & + & + \\
\hline B 27 & toruloides & + & $\mathrm{v}$ & + \\
\hline B 28 & & + & $\mathrm{v}$ & + \\
\hline B 29 & & + & $\mathrm{v}$ & + \\
\hline B 30 & Sporid. johansonii & + & $\mathrm{v}$ & + \\
\hline B 31 & pararoseus & + & $\mathrm{v}$ & + \\
\hline B 32 & ruinenii & + & + & + \\
\hline B 33 & salmonicolor & + & $\mathrm{v}$ & + \\
\hline
\end{tabular}

+ , positive; w, weak; v, less definite; - , negative.

On the other hand, the alkaline-ethanol-DBB test described by HAGLER and AHEARN (6) required the exact preparation of DBB reagent and culture conditions and its procedures are very complicated. As for the results of a simple procedure on solid medium and alkaline-ethanol procedure, they often gave less definitive results. In this study, we observed indecisive coloration in 35 strains belonging to 21 species of Cystofilobasidium, Rhodosporidium, Sporidiobolus, Bullera, Rhodotorula, and Sporobolomyces. Generally, extracellular urease production is common in basidiomycetous yeasts. However, it has been reported that some ascomycetous yeasts also produce urease. In this study, urease activities were observed in several 
Table 4. The occurrence of pyrophosphatidic acid, DBB reaction and urease activity in imperfect yeasts.

\begin{tabular}{|c|c|c|c|c|}
\hline Strain No. & Yeasts & Pyro-PA & DBB & Urease \\
\hline I 01 & Br. abstinens & - & - & - \\
\hline I 02 & anomalus & - & _- & - \\
\hline I 03 & bruxellensis & - & - & - \\
\hline I 04 & claussenii & - & - & - \\
\hline I 05 & custersianus & - & - & - \\
\hline I 06 & intermedius & - & - & - \\
\hline I 07 & lambicus & - & - & - \\
\hline I 08 & naardenensis & - & - & - \\
\hline I 09 & Kl. africana & - & - & - \\
\hline I 10 & & - & - & - \\
\hline I 11 & & - & - & - \\
\hline I 12 & apiculata & - & _- & - \\
\hline I 13 & & - & - & - \\
\hline I 14 & & - & - & - \\
\hline I 15 & corticis & - & - & - \\
\hline I 16 & & - & - & - \\
\hline I 17 & & - & - & - \\
\hline I 18 & & - & _- & - \\
\hline I 19 & & - & - & - \\
\hline I 20 & japonica & - & - & - \\
\hline I 21 & & - & - & - \\
\hline I 22 & javanica & - & - & - \\
\hline I 23 & My. melibiosi & - & _- & - \\
\hline I 24 & mucilagina & - & - & - \\
\hline I 25 & O. margaritiferum & - & - & - \\
\hline I 26 & Sait. complicata & - & - & + \\
\hline I 27 & & - & - & + \\
\hline I 28 & Trig. variabilis & - & _- & - \\
\hline I 31 & B. alba & + & - & - \\
\hline I 32 & & + & _- & - \\
\hline I 33 & & + & - & - \\
\hline I 34 & & + & - & - \\
\hline I 35 & dendrophila & + & + & + \\
\hline I 36 & piricola & + & + & + \\
\hline I 37 & singularis & + & + & + \\
\hline I 38 & tsugae & $+w$ & $\mathrm{v}$ & $\mathrm{v}$ \\
\hline I 39 & Cr. albidus var. albidus & + & + & + \\
\hline I 40 & var. aerius & + & + & + \\
\hline I 41 & ater & + & + & + \\
\hline I 42 & bhutanensis & + & + & $\mathrm{v}$ \\
\hline I 43 & gastricus & + & + & + \\
\hline I 44 & heveanensis & + & + & + \\
\hline I 45 & himalayensis & + & + & + \\
\hline I 46 & kuetzingii & + & + & + \\
\hline I 47 & laurentii & + & + & + \\
\hline I 48 & luteolus & + & + & + \\
\hline I 49 & macerans & + & + & + \\
\hline I 51 & neoformans & + & + & + \\
\hline
\end{tabular}


Table 4. (continued)

\begin{tabular}{|c|c|c|c|c|}
\hline Strain No. & Yeasts & Pyro-PA & DBB & Urease \\
\hline I 52 & Cr. skinneri & + & + & + \\
\hline I 53 & terreus & + & + & + \\
\hline I 54 & Ph. rhodozyma & + & + & + \\
\hline I 55 & Rh. araucariae & + & $\mathrm{v}$ & + \\
\hline I 56 & aurantiaca & + & $\mathrm{v}$ & + \\
\hline I 57 & fujisanensis & + & + & + \\
\hline I 58 & & + & + & + \\
\hline I 59 & glutinis & + & $\mathrm{v}$ & + \\
\hline I 60 & & + & $\mathrm{v}$ & + \\
\hline I 61 & & + & $\mathrm{v}$ & + \\
\hline I 62 & & + & $\mathrm{v}$ & + \\
\hline I 63 & & + & $\mathrm{v}$ & + \\
\hline I 64 & & + & $\mathrm{v}$ & + \\
\hline I 65 & & + & $\mathrm{v}$ & + \\
\hline I 66 & & + & $\mathrm{v}$ & + \\
\hline I 67 & glutinis var. salinaria & + & + & + \\
\hline I 68 & graminis & + & $\mathrm{v}$ & + \\
\hline I 69 & & + & $\mathrm{v}$ & + \\
\hline I 70 & lactosa & + & + & + \\
\hline I 71 & minuta & + & + & + \\
\hline I 72 & & + & $\mathrm{v}$ & + \\
\hline I 73 & rubra & + & $\mathrm{v}$ & + \\
\hline I 74 & Sp. albo-rubescens & + & + & + \\
\hline I 75 & gracilis & + & + & + \\
\hline I 76 & hispanicus & + & $\mathrm{v}$ & + \\
\hline I 77 & odorus & + & $\mathrm{v}$ & + \\
\hline I 78 & roseus & + & + & + \\
\hline I 79 & & + & $\mathrm{v}$ & + \\
\hline I 80 & & + & + & + \\
\hline I 81 & & + & + & + \\
\hline I 82 & & + & $\mathrm{v}$ & + \\
\hline I 84 & salmonicolor & + & $\mathrm{v}$ & + \\
\hline I 85 & shibatanus & + & + & + \\
\hline I 86 & & + & $\mathrm{v}$ & + \\
\hline I 87 & St. elviae & + & + & + \\
\hline
\end{tabular}

+ , positive; w, weak; v, less definite; -, negative.

ascomycetous species: Cit. matritensis (A 02), I. terricola (A 12), L. starkeyi (A 14), 2 strains (A 17 and A 18) of Nadsonia, 3 (A 27, A 28 and A 29) of Schizosaccharomyces, 2 (I 26 and I 27) of Saitoella complicata, and Yarrowia lipolytica (A 34). In contrast, urease activity was indicated very faintly in several basidiomycetous yeasts: 2 strains (B 01 and B 02) of Cystof. capitatum, 1 (B 18) of Rhodosp. diobovatum, 1 (B 20) of Rhodosp. infirmominiatum, 2 (B 23 and B 24) of Rhodosp. malvinellum, 1 (I 38) of B. tsugae, and 1 (I 42) of Cr. bhutanensis. Therefore, the urease test does not reliably discriminate between ascomycetous and basidiomycetous species. 
Table 5. The occurrence of pyrophosphatidic acid and DBB reaction, urease activity, and fermentation in Candida.

\begin{tabular}{|c|c|c|c|c|c|}
\hline Strain No. & Species & Pyro-PA & DBB & Urease & Fermentation \\
\hline C 01 & C. acutus & + & + & + & - \\
\hline $\mathrm{C} 03$ & amylolenta & + & + & + & - \\
\hline C 04 & antarctica & + & + & + & - \\
\hline C 05 & aquatica & + & + & + & - \\
\hline C 06 & auriculariae & + & + & + & - \\
\hline C 08 & bacarum & + & + & + & - \\
\hline C 16 & curiosa & + & + & + & + \\
\hline C 17 & curvata & + & + & + & - \\
\hline C 18 & diffloens & + & + & + & - \\
\hline C 19 & & + & + & + & - \\
\hline C 24 & foliorum & + & $\mathrm{v}$ & + & - \\
\hline C 25 & fusiformata & + & $\mathrm{v}$ & + & - \\
\hline C 28 & humicola & + & + & + & - \\
\hline C 29 & & + & + & + & - \\
\hline C 30 & & w & + & + & - \\
\hline C 32 & hylophila & + & + & + & - \\
\hline C 33 & ingeniosa & + & + & + & - \\
\hline C 36 & javanica & + & w & + & - \\
\hline C 46 & muscorum & + & + & + & - \\
\hline C 49 & philyla & + & + & + & - \\
\hline C 50 & podzolica & + & + & + & - \\
\hline C 52 & pustula & + & + & + & - \\
\hline C 55 & sonkii & + & + & $\mathrm{v}$ & - \\
\hline C 61 & tsukubaensis & + & + & + & - \\
\hline C 02 & albicans & + & - & - & - \\
\hline C 07 & auriculariae & - & - & - & + \\
\hline C 09 & blankii & - & - & - & w \\
\hline C 10 & boidinii & - & - & - & + \\
\hline C 11 & bombicola & - & - & - & + \\
\hline C 12 & buffonii & - & - & $\mathrm{v}$ & + \\
\hline C 13 & catenulata & - & - & - & w \\
\hline C 14 & chiropterorum & - & - & - & - \\
\hline C 15 & colliculosa & - & - & - & + \\
\hline C 20 & edax & - & - & - & - \\
\hline C 21 & famata & - & - & - & + \\
\hline C 22 & & - & - & - & + \\
\hline C 23 & & - & - & - & + \\
\hline C 26 & guilliermondii & - & - & - & + \\
\hline C 27 & hellenica & - & - & - & + \\
\hline C 31 & hydrocarbofumaria & - & - & - & - \\
\hline C 34 & inositophila & - & - & - & + \\
\hline C 35 & intermedia & - & - & - & + \\
\hline C 37 & krissii & - & - & - & $-1+$ \\
\hline C 38 & kruisii & - & - & - & + \\
\hline C 39 & krusei & - & - & $\mathrm{w}$ & + \\
\hline C 40 & lambica & - & - & - & + \\
\hline C 41 & lusitaniae & - & - & - & + \\
\hline C 42 & magnoliae & - & - & - & + \\
\hline
\end{tabular}


Table 5. (continued)

\begin{tabular}{clcccc}
\hline Strain No. & Species & Pyro-PA & DBB & Urease & Fermentation \\
\hline C 43 & C. maltosa & - & - & - & + \\
C 44 & mamillae & - & - & - & + \\
C 45 & mucilagina & - & - & - & - \\
C 47 & parapsilosis & - & - & - & + \\
C 48 & psychrophila & - & - & - & + \\
C 51 & rugosa & - & - & - & - \\
C 53 & sake & - & - & - & - \\
C 54 & steatolytica & - & - & - & + \\
C 56 & stellata & - & - & - & + \\
C 57 & terebra & - & - & - & + \\
C 58 & tropicalis & - & - & - & + \\
C 59 & & - & - & - & + \\
C 60 & utilis & - & - & - & + \\
C 62 & valdiviana & - & - & - & + \\
C 63 & valida & - & - & - & + \\
C 64 & & - & - & - & + \\
C 65 & versatilis & - & - & - & + \\
C 66 & zeylanoides & - & - & - & + \\
C 67 & & - & - & - & + \\
\hline
\end{tabular}

+ , positive; w, weak; v, less definite; - , negative.

\section{Presence of pyro-PA in genus Candida}

For the genus Candida, the results of pyro-PA, DBB, urease, and fermentation tests are shown in Table 5. Pyro-PA was detected in 19 species (22 strains) that were assumed to be basidiomycetous by MeYER et al. (24), and in C. acutus (C 01) and $C$. fusiformata (C 25). C. acutus was described as a hemibasidiomycetous species by Goto (25) and $C$. fusiformata was reported as being a species related to Rhodotorula and Cryptococcus (26). In contrast, no pyro-PA was detected in 43 strains of 38 ascomycetous species. Exceptionally, pyro-PA was found in C. albicans (C 02) which was considered to be an ascomycetous species. The DBB test in 19 basidiomycetous species (22 strains) in which pyro-PA was found were positive, although coloration was less definitive in C. foliorum (C 24) and C. fusiformata (C 25). Twenty basidiomycetous yeasts (23 strains) in which pyro-PA was detected, produced urease, although urease activity was indefinite in $C$. sonckii (C 55). Twenty basidiomycetous species (23 strains), except $C$. curiosa (C 16), did not ferment glucose. As in the above results, we observed that the DBB and urease tests gave less definitive results for several species of Candida. Therefore, basidiomycetous species in the genus Candida can be discriminated correctly by the pyro-PA test, in addition to the DBB, urease, and fermentation tests as a practical procedure. C. albicans (C 02), the ascomycetous species in which pyro-PA was found may require additional studies to elucidate this presence of pyro-PA. 
Table 6. The occurrence of pyrophosphatidic acid and DBB reaction, urease activity, and fermentation in Trichosporon.

\begin{tabular}{cccccc}
\hline Strain No. & Species & Pyro-PA & DBB & Urease & Fermentation \\
\hline T 01 & Tr. aculeatum & - & - & - & $-/ \mathrm{w}$ \\
T 02 & & - & - & - & $-/ \mathrm{w}$ \\
T 05 & capitatum & - & - & - & - \\
T 06 & & - & - & - & - \\
T 16 & fennicum & - & - & - & + \\
T 17 & fermentans & - & - & - & + \\
T 18 & & - & - & - & + \\
T 19 & & - & - & - & + \\
T 20 & melibiosaceum & - & - & - & + \\
T 03 & aquatile & + & + & + & - \\
T 04 & brassicae & + & + & + & - \\
T 07 & cutaneum & + & + & + & - \\
T 08 & & + & + & + & - \\
T 09 & & + & + & + & - \\
T 10 & & + & + & + & - \\
T 11 & & + & + & + & - \\
T 12 & & + & + & + & - \\
T 13 & & + & + & + & - \\
T 14 & & + & + & + & - \\
T 15 & & + & + & + & - \\
T 21 & pullulans & + & + & + & - \\
T 22 & & + & + & + & - \\
T 23 & & + & + & + & - \\
T 24 & & + & + & + & - \\
\hline
\end{tabular}

+ , positive; w, weak; - , negative.

Presence of pyro-PA in the genus Trichosporon

For the genus Trichosporon, the results of the pyro-PA, DBB, urease and fermentation tests are shown in Table 6. Pyro-PA was found in a total of 15 strains of 4 species ( $T r$. aquatile, $T r$. brassicae, $\operatorname{Tr}$. cutaneum, and $\operatorname{Tr}$. pullulans) that were assumed to be basidiomycetous by KREGER-VAN RIJ (27). On the other hand, pyroPA was not detected in 9 strains of 5 species ( 2 strains of Tr. aculeatum, 2 of $T r$. capitatum, 1 of $T r$. fennicum, 3 of Tr. fermentans, and 1 of $T r$. melibiosaceum) that were classified as ascomycetous species by KREGER-VAN RIJ (27). For basidiomycetous species in which pyro-PA was found, the DBB and urease tests were positive and the fermentation test was negative, without exception. On the contrary, for ascomycetous species in which pyro-PA was not detected, the results of DBB and urease tests were negative without exception. Some authors have proposed to split the genus Trichosporon(28-30). According to these authors Trichosporon can be separated into the basidiomycetous and ascomycetous species based on the cell wall and septa structures, DBB reaction, urease activity, composition of the carbohydrate of the cells, and the GC content of DNA.

The results of pyro-PA, DBB, and urease tests in ascomycetous and ba- 
Table 7. The occurrence of pyrophosphatidic acid, DBB reaction, and urease activity in yeast genera.

\begin{tabular}{|c|c|c|c|c|c|c|}
\hline Yeasts & Genera & Species & Strains & Pyro-PA & DBB & Urease \\
\hline \multicolumn{7}{|l|}{ Ascomycetous } \\
\hline Teleomorph & 23 & 28 & 34 & - & - & - \\
\hline \multicolumn{7}{|l|}{ Anamorph } \\
\hline Brettanomyces & & 8 & 8 & - & - & - \\
\hline Kloeckera & & 5 & 14 & - & - & - \\
\hline Mухогута & & 2 & 2 & - & - & - \\
\hline Oosporidium & & 1 & 1 & - & - & - \\
\hline Saitoella & & 1 & 2 & - & - & - \\
\hline Trigonopsis & & 1 & 1 & - & - & - \\
\hline Candida (A) & & 38 & 43 & - & - & - \\
\hline Trichosporon (A) & & 5 & 9 & - & - & - \\
\hline \multicolumn{7}{|l|}{ Basidiomycetous } \\
\hline \multicolumn{7}{|l|}{ Teleomorph } \\
\hline Cystofilobasidium & & 1 & 2 & + & $\mathrm{v}$ & $+/ \mathrm{w}$ \\
\hline Filobasidiella & & 1 & 2 & + & + & + \\
\hline Filobasidium & & 3 & 3 & + & + & + \\
\hline Leucosporidium & & 3 & 3 & + & + & + \\
\hline Rhodosporidium & & 6 & 19 & + & $+/ \mathrm{v}$ & $+/ \mathrm{w} /-$ \\
\hline Sporidiobolus & & 4 & 4 & + & $+/ \mathrm{v}$ & $+/ w$ \\
\hline \multicolumn{7}{|l|}{ Anamorph } \\
\hline Bullera & & 5 & 8 & + & $+/ \mathrm{v}$ & $+/ \mathrm{w}$ \\
\hline Cryptococcus & & 13 & 14 & + & + & + \\
\hline Phaffia & & 1 & 1 & + & + & + \\
\hline Rhodotorula & & 8 & 19 & + & $+/ \mathrm{v}$ & $+/ \mathrm{w}$ \\
\hline Sporobolomyces & & 7 & 12 & + & $+/ \mathrm{v}$ & $+/ \mathrm{w}$ \\
\hline Sterigmatomyces & & 1 & 1 & + & + & + \\
\hline Candida (B) & & 21 & 24 & + & $+/ \mathrm{v}, \mathrm{w}$ & $+/ \mathrm{v}$ \\
\hline Trichosporon (B) & & 4 & 15 & + & + & + \\
\hline
\end{tabular}

+ , positive; w, weak; v, less definite; -, negative.

sidiomycetous yeast genera are shown in Table 7. Generally, for the yeast species (or genera) in which pyro-PA was detected, the DBB reaction and urease activity were positive. The species (or genera) having those characteristics belong to the basidiomycetous yeasts. In contrast, for the species (or genera) in which pyro-PA was not found, DBB coloration and urease activity were usually negative. The species (or genera) having those characteristics belong to the ascomycetous yeasts. When the pyro-PA test is compared with both the DBB and urease tests, it is a comparatively simple procedure and the presence of pyro-PA is detected without difficulty. We consider that discriminating ascomycetous from basidiomycetous yeast can be done more distinctly by using the pyro-PA tests. Therefore, this test is recommended as a routine and practical procedure for discriminating between ascomycetous and basidiomycetous yeast species or genera. 
We thank Prof. Dr. K. Komagata, Institute of Applied Microbiology, The University of Tokyo, Japan and Prof. Dr. T. Sutou, Nippon Veterinary and Zootechnical College, Musashino, Tokyo, Japan, for supplying yeast strains.

\section{REFERENCES}

1) N. J. W. Kreger-Van RiJ, J. Gen. Microbiol., 68, 87 (1971).

2) J. L. Gay and M. Martin, Arch. Microbiol., 78, 145 (1971).

3) S. BaharaEen and H. S. VishniaC, Mycologia, 73, 618 (1981).

4) R. B. Simonons and D. G. Ahearn, Mycologia, 79, 38 (1987).

5) J. P. Van Der Walt and V. K. Hopsu-Havu, Antonie van Leeuwenhoek J. Microbiol. Serol., 42, 157 (1976).

6) A. H. Hagler and D. G. Ahearn, Int. J. Syst. Bacteriol., 31, 204 (1986).

7) J. Cazin, Jr., T. R. Kozel, D. M. Lupan, and W. R. Burt, J. Bacteriol., 100, 760 (1969).

8) K. Sen and K. Komagata, J. Gen. Appl. Microbiol., 25, 127 (1979).

9) T. NaKase and K. Komagata, J. Gen. Appl. Microbiol., 17, 363 (1971).

10) T. NAKASE, Proc. IV IFS: Ferment. Technol. Today, 785 (1972).

11) J. A. Barnett, R. W. Payne, and D. Yarrow, Yeasts, Characteristics and Identification, Cambridge Univ. Press, Cambridge (1983), p. 811.

12) N. J. W. Kreger-Van RiJ (ed.), The Yeasts, a Taxonomic Study, 3rd Rev. and Enlarged ed., Elsevier, Amsterdam (1984), p. 1083.

13) T. ItoH and H. KaneKo, J. Biochem., 75, 1291 (1974).

14) T. ITOH and H. KaneKo, J. Biochem., 78, 817 (1975).

15) H. Kaneko, M. Hosohara, M. Yanaka, and T. Iтoh, Lipids, 11, 837 (1976).

16) T. Itoh and H. KaneKo, Yukagaku, 23, 350 (1974).

17) J. Folch, M. Lees, and G. H. Sloane-Stanley, J. Biol. Chem., 226, 497 (1957).

18) W. I. Golubev, V. M. Blagodatskaya, S. O. Suetin, and R. S. Trotstenko, Int. J. System. Bacteriol., 31, 91 (1981).

19) J. Lodder (ed.), The Yeasts, a Taxonomic Study, 2nd ed., North-Holland Publ. Co., Amsterdam (1970), p. 1385

20) J. P. Van Der Walt, A. C. M. Weijman, and J. A. Vonarx, Sydowia, Ann. Mycol. Ser. II, 34, 191 (1981).

21) L. Rodrigues de Miranda, Antonie van Leeuwenhoek J. Microbiol. Serol., 44, 439 (1978).

22) S. Goto, J. Sugiyama, M. Намамото, and K. Komagata, J. Gen. Appl. Microbiol., 33, 75 (1987).

23) S. Goto and J. Sugiyama, Can. J. Bot., 48, 2097 (1970).

24) S. A. Meyer, D. G. Ahearn, and D. Yarrow, Genus Candida Berkhout, In The Yeasts, a Taxonomic Study, 3rd ed., ed. by N. J. W. KREGER-VAN RIJ, 3rd Rev. and Enlarged ed., Elsevier, Amsterdam (1984), p. 585.

25) S. Goto, J. Gen. Appl. Microbiol., 25, 145 (1979).

26) R. W. M. Buhagiar, J. Gen. Microbiol., 110, 9 (1979).

27) N. J. W. Kreger-Van RiJ, Genus Trichosporon Behrend, In The Yeasts, a Taxonomic Study, 3rd ed., ed. by N. J. W. Kreger-VAN RiJ, 3rd Rev. and Enlarged ed., Elsevier, Amsterdam (1984), p. 933.

28) A. Kokova-Kratochvilova, E. Slavikova, J. ZemeK, and L. Kuniak, Proc. 5th Int. Spec. Symposium Yeasts (1977), p. 9.

29) A. C. M. Weijman, Antonie van Leeuwenhoek J. Microbiol., 45, 119 (1979).

$30)$ E. Gueho, J. Tredick, and H. J. Phaff, Antonie van Leeuwenhoek J. Microbiol., 50, 17 (1984). 